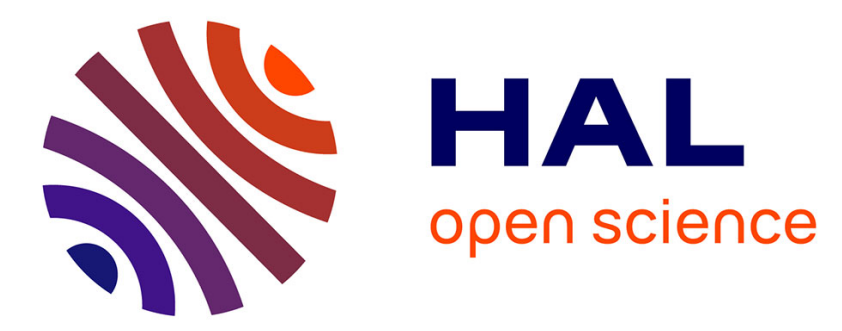

\title{
Stationary gravity waves with the zero mean vorticity in stratified fluid
}

Didier Clamond, Yury Stepanyants

\section{To cite this version:}

Didier Clamond, Yury Stepanyants. Stationary gravity waves with the zero mean vorticity in stratified fluid. 2011. hal-00566211

\section{HAL Id: hal-00566211 \\ https://hal.science/hal-00566211}

Submitted on 15 Feb 2011

HAL is a multi-disciplinary open access archive for the deposit and dissemination of scientific research documents, whether they are published or not. The documents may come from teaching and research institutions in France or abroad, or from public or private research centers.
L'archive ouverte pluridisciplinaire $\mathbf{H A L}$, est destinée au dépôt et à la diffusion de documents scientifiques de niveau recherche, publiés ou non, émanant des établissements d'enseignement et de recherche français ou étrangers, des laboratoires publics ou privés. 


\title{
Stationary gravity waves with the zero mean vorticity in stratified fluid
}

\author{
Didier CLAMOND ${ }^{1 *}$ and Yury STEPANYANTS ${ }^{2 * *}$ \\ ${ }^{1}$ Université de Nice - Sophia Antipolis, Laboratoire J.-A. Dieudonné, Parc Valrose, \\ 06108 Nice cedex 2, France. E-mail: didierc@unice.fr \\ ${ }^{2}$ University of Southern Queensland, Department of Mathematics and Computing, \\ Toowoomba, Australia. E-mail: yuas50@gmail.com
}

\begin{abstract}
A new approach to the description of stationary plane waves in ideal density stratified incompressible fluid is considered without the application of Boussinesq approximation. The approach is based on the equation derived by Dubreil-Jacotin $[4,5,6]$ and Long [7] with the additional assumption that the mean vorticity of the flow is zero. It is shown that in the linear approximation the spectrum of eigenmodes and dispersion equations corresponding to these eigenmodes can be found in the closed analytical forms for many particular relationships between the fluid density and stream function. Examples are presented for waves of infinitesimal amplitude. Exact expression for the velocity of solitary wave of any amplitude is derived.
\end{abstract}

Key words: Stratified fluid; perfect fluid; incompressible flow; zero mean vorticity; surface waves; internal waves; solitary waves; boundary-value problem; periodic solutions.

*) Corresponding author.

**) This work was undertaken within the framework of two short visitorship programs in February 2009 and January 2011, when Y. Stepanyants visited the Laboratory J.-A. Dieudonné of the University of Nice. Y.S. is highly appreciated the Laboratory staff for the invitations and hospitality. 


\section{Introduction}

The problem of analytical description of surface and internal waves in density stratified fluid is still topical both from the academic and practical point of views. Practical interest to the problem is conditioned by its application mainly in the physical oceanography, although other applications are also possible. Analytical methods of investigation of water wave structure are traditionally based on the consideration of the cases when the fluid is either exponentially stratified, so that the buoyancy parameter (alias the Brunt-Väisälä frequency, see below), is constant or when the fluid stratification can be modeled by a few numbers of homogeneous layers. For the problem simplification, it is also traditionally used Boussinesq approximation within the framework of which the fluid density $\rho(y)$ is treated as a constant, $\rho(y) \approx \rho_{0}$, everywhere in the governing equations, except those terms which contain the gravity acceleration $g$ as the multiplier (see, e.g., $[1,2,3]$ ). Formally mathematically, this approximation corresponds to the limit when $\mathrm{d} \rho / \mathrm{d} y \rightarrow 0, g \rightarrow \infty$, whereas $g \mathrm{~d} \rho / \mathrm{d} y=$ Cst. In other cases, the numerical methods are usually applied to construct solutions describing a structure of linear and nonlinear waves. But, even in the cases when the problem is studied by means of numerical methods, Boussinesq's approximation is widely used.

In the meantime, the development of rigorous analytical methods for the description of water wave structure still remains topical as this allows one to gain an insight about the peculiarities of water waves in density stratified fluids and obtain some benchmark solutions for testing numerical results. In this paper we present an attempt to develop a method of rigorous description of surface and internal waves in density stratified fluid beyond the Boussinesq approximation. Our approach is based on the exploitation of the well-known Dubreil-Jacotin-Long (DJL) equation $[4,5,6,7]$ relating the fluid density $\rho$ and stream function $\psi$ (see below). It is shown that in several particular case of the relationship between these two quantities, the DJL equation can be solved analytically, at least, in the linear approximation in the wave amplitude. We define the class of wave motions with the zero mean vorticity (ZMV) and investigate the boundary-value problem for the waves of infinitesimal amplitude for various dependences $\rho(\psi)$. The most detailed results are obtained, in particular, for the linear dependence $\rho(\psi)$. The dispersion relation and mode structure are obtained in the shallow- and deep-water limits beyond the Boussinesq approximation. We show also that the velocity of a finite amplitude solitary wave can be deduced in the closed form for the wave of arbitrary amplitude.

The paper is organized as follows. For the easy self-contained reference, the hypothesis, notations and derivations are detailed in section 2. Further details can be found in the Appendix A. Peculiar types of stratifications and some parameters are introduced in section 3. Main results are derived in sections 4-9. In the Conclusion, we summarize and discuss the results obtained.

\section{Formulation of the problem}

In this section, we present the main hypothesis and equations of motion. Further technical details can be found in the Appendix A. 


\subsection{Basic equations}

Let us consider a two-dimensional steady wave motion in a perfect incompressible fluid of density $\rho>0$ which may vary in the vertical direction (density stratified fluid). The unperturbed fluid depth $h$ is assumed constant $(0<h \leqslant \infty)$, the surface tension is neglected in this study, and $g$ denotes the acceleration due to gravity. Let $x$ and $y$ be the horizontal and vertical coordinates, respectively, as shown in the sketch of Fig. 1. Denote by $y=\eta(x)$ the shape of the free surface perturbed by a wave; in the absence of wave, the unperturbed water surface is described by equation $y=0$, whereas $y=-h$ denotes the impermeable horizontal bottom. The wave propagates toward the positive $x$-direction if the phase velocity $c$ is positive in the immovable (reference) coordinate frame.

The condition of fluid incompressibility - viz. $\boldsymbol{\nabla} \cdot \boldsymbol{u}=0$, where $\boldsymbol{u}=(u, v)$ is the velocity vector and $\nabla$ is the gradient operator - allows us to introduce a stream-function $\psi$ such that the horizontal and vertical velocity components, $u$ and $v$ respectively, can be presented as $u=\partial \psi / \partial y$ and $v=-\partial \psi / \partial x$. Since the flow is assumed steady, the bottom and the free surface are stream lines; let us denote the corresponding constant values of these streamlines as $\psi_{\mathrm{b}} \equiv \psi(y=-h)$ and $\psi_{\mathrm{s}} \equiv \psi(y=\eta)$. Without loss of generality, we can impose arbitrarily the value of one of these constants $\left(\psi_{\mathrm{b}}\right.$ or $\left.\psi_{\mathrm{s}}\right)$; this determines the function $\psi$ univocally (a convenient choice will be taken below). Finally, by $P$ we denote the pressure, which is assumed to be zero at the free surface, and by $\omega=\partial v / \partial x-\partial u / \partial y=-\nabla^{2} \psi$ we denote the flow vorticity, the only nonzero component of which is directed perpendicular to the $x y$-plane.

For incompressible fluids, the mass conservation equation yields $\mathrm{D} \rho / \mathrm{D} t=0$, where $\mathrm{D} / \mathrm{D} t \equiv$ $\partial / \partial t+\boldsymbol{u} \cdot \boldsymbol{\nabla}$ is the traditional notation of the substantial derivative (here $\mathrm{D} / \mathrm{D} t=\boldsymbol{u} \cdot \boldsymbol{\nabla}$ because the flow is steady). As follows from this equation, $\rho$ is constant along the trajectory of a fluid particle. Hence, for a steady flow the streamlines are simply coincide with the particle trajectories and thus

$$
\rho=\rho(\psi) .
$$

Note that this relation is not revertible in general, i.e., it does not necessarily implies that $\psi=\psi(\rho)$. For example, in the homogeneous fluid $\rho$ is constant in the all space occupied by fluid, whereas $\psi$ may vary.

The Euler momentum equation for the incompressible fluid can be written as

$$
\rho \frac{\mathrm{D} \boldsymbol{u}}{\mathrm{D} t}=-\nabla(P+\rho g y)+g y \nabla \rho .
$$

After scalar multiplication of this equation by $\boldsymbol{u}$, exploiting the relations $\boldsymbol{u} \cdot \nabla \rho=\mathrm{D} \rho / \mathrm{D} t=0$, $\boldsymbol{u} \cdot \boldsymbol{\nabla}(P+\rho g y)=\mathrm{D}(P+\rho g y) / \mathrm{D} t$ and $\rho \boldsymbol{u} \cdot \mathrm{D} \boldsymbol{u} / \mathrm{D} t=\rho \mathrm{D}\left(|\boldsymbol{u}|^{2} / 2\right) / \mathrm{D} t=\mathrm{D}\left(\rho|\boldsymbol{u}|^{2} / 2\right) / \mathrm{D} t$, we obtain after some elementary algebra

$$
\frac{\mathrm{D}}{\mathrm{D} t}\left[\rho \frac{|\boldsymbol{u}|^{2}}{2}+P+\rho g y\right]=0 .
$$

This implies that the expression in the brackets is constant along each stream function:

$$
\frac{P}{\rho}+g y+\frac{u^{2}+v^{2}}{2}=B(\psi)
$$


where $B(\psi)$ is the Bernoulli integral (see, e.g., [2]).

Substituting Eqs. (1) and (3) into the $y$-component of the Euler equation (2), one readily obtains the equality

$$
\frac{P}{\rho^{2}} \frac{\mathrm{d} \rho}{\mathrm{d} \psi}+\frac{\mathrm{d} B}{\mathrm{~d} \psi}=-\omega .
$$

We emphasize that in Eqs. (1)-(4) the quantities $\rho$ and $B$ are functions of $\psi$ solely, while the stream function $\psi$, as well as other quantities, $u, v, \omega$ and $P$, depend of both spatial coordinates $x$ and $y$ and time $t$. Eliminating $P$ between (3) and (4), we obtain the wellknown DJL equation $[4,5,6,7]$ :

$$
\rho \nabla^{2} \psi+\frac{1}{2} \frac{\mathrm{d} \rho}{\mathrm{d} \psi}\left[(\nabla \psi)^{2}+2 g y\right]=\frac{\mathrm{d}(\rho B)}{\mathrm{d} \psi} .
$$

Let us consider a periodic solution to this equation with the spatial period $\lambda=2 \pi / k$, where $k$ is a wave number. The period of a solution may be infinite, in particular; in this case the corresponding solution describes a non-periodic wave.

Let us define the zero water level as the perturbation of the water surface $\eta$ averaged over the spatial period $\lambda$ :

$$
\langle\eta\rangle \equiv \frac{k}{2 \pi} \int_{-\pi / k}^{\pi / k} \eta(x) \mathrm{d} x=0 .
$$

Similarly, we define by $-c$ the mean horizontal velocity of the fluid in the coordinate frame related with the stationary wave with the velocity $c$ (moving to the right if $c>0$ ):

$$
c \equiv-\frac{k}{2 \pi h} \int_{-\pi / k}^{\pi / k} \int_{-h}^{\eta} u(x, y) \mathrm{d} y \mathrm{~d} x=\frac{\psi_{\mathrm{b}}-\psi_{\mathrm{s}}}{h} .
$$

With the condition (6) and zero pressure at the water surface, Eq. (3) defines the Bernoulli integral at the free surface:

$$
B_{\mathrm{s}} \equiv B\left(\psi_{\mathrm{s}}\right)=\frac{\left\langle u_{\mathrm{s}}^{2}+v_{\mathrm{s}}^{2}\right\rangle}{2}=\frac{k}{4 \pi} \int_{-\pi / k}^{\pi / k}\left[(\nabla \psi)^{2}\right]_{y=\eta} \mathrm{d} x .
$$

The definition of $B$ for $\psi \neq \psi_{\text {s }}$ requires a further consideration.

\subsection{Wave motions with the zero mean vorticity}

Note first that if $\omega=0$, then Eq. (4) implies that either $\rho$ and $B$ are constants (this corresponds to homogeneous fluid), or $P$ is the specific function of $\psi$, namely:

$$
P(\psi)=-\rho^{2} \frac{\mathrm{d} B}{\mathrm{~d} \psi}\left(\frac{\mathrm{d} \rho}{\mathrm{d} \psi}\right)^{-1},
$$


(this corresponds to isobaric streamlines). But, as has been proven by Dubreil-Jacotin [5], the only wave motion with the isobaric streamlines is Gerstner wave [9] (see also [10]), which is, however, rotational, i.e. with $\omega(\psi) \neq 0$. Hence, any wave motion of density stratified fluid with $\mathrm{d} \rho / \mathrm{d} \psi \neq 0$ is necessarily rotational.

In general, the vorticity associated with the wave can co-exist with the ambient vorticity of the mean flow. In this paper we focus, however, on the particular case of such motions when the mean-flow vorticity is zero. In addition to that, we assume that the mean vorticity associated with the wave motion is also zero, i.e. $\langle\omega\rangle=0$. Here, the averaging integral is calculated over the spatial period on $x$ along each constant streamline $\psi$. We call such special flow the zero-mean vorticity (ZMV) flow. Notice that Gerstner wave does not belong to this class of wave motion; the mean vorticity of such wave is nonzero.

For wave motion with the zero mean vorticity, Eq. (4) after the averaging along the constant streamline yields the equation for the mean pressure:

$$
\langle P\rangle=-\rho^{2} \frac{\mathrm{d} B}{\mathrm{~d} \psi}\left(\frac{\mathrm{d} \rho}{\mathrm{d} \psi}\right)^{-1},
$$

provided that $\mathrm{d} \rho / \mathrm{d} \psi \neq 0$. Averaging now the DJL equation (5) over the wave period, we also obtain (see Appendix A for details):

$$
\frac{\mathrm{d}(\rho B)}{\mathrm{d} \psi}=\left[B_{\mathrm{s}}+\frac{g\left(\psi_{\mathrm{s}}-\psi\right)}{c}\right] \frac{\mathrm{d} \rho}{\mathrm{d} \psi} .
$$

This completes the definition of the Bernoulli integral for the ZMV-flows and allows us to present the DJL equation (5) in the form:

$$
\nabla^{2} \psi+\frac{1}{\rho} \frac{\mathrm{d} \rho}{\mathrm{d} \psi}\left[\frac{1}{2}(\nabla \psi)^{2}-B_{\mathrm{s}}+(g / c)\left(\psi-\psi_{\mathrm{s}}\right)+g y\right]=0 .
$$

This equation has to be augmented by the bottom and surface boundary conditions to complete the problem statement. This will be done further. Assuming then a specific dependence of $\rho(\psi)$ we will investigate the resultant equations.

Notice that Brown and Christie has stated [11] that in application to solitary waves Eq. (12) remains valid not only when all streamlines go to infinity, but when they are closed and confined within a certain space domain too. In the latter case, the corresponding solitary waves are strongly nonlinear representing flows with compact vortex cores.

\subsection{Remarks}

(i) For solitary waves, it is often assumed (see, e.g., [11, 12]) that the fluid flow is uniform at the infinity. However, since we are dealing here with the periodic waves, in general, the upstream condition of uniform flow is replaced by the condition of the ZMV. The latter condition reduces to the former one for solitary waves.

(ii) After multiplication of Eq. (5) by $\left(\rho_{0} / \rho\right)^{\frac{1}{2}}$, where $\rho_{0}>0$ is a constant reference density, and introducing new dependent function

$$
\Psi(\psi) \equiv \int\left(\frac{\rho}{\rho_{0}}\right)^{\frac{1}{2}} \mathrm{~d} \psi \quad \Longleftrightarrow \quad \psi(\Psi)=\int\left(\frac{\rho_{0}}{\rho}\right)^{\frac{1}{2}} \mathrm{~d} \Psi,
$$


Eq. (5) reduces to

$$
\rho_{0} \nabla^{2} \Psi+\frac{\mathrm{d} \rho}{\mathrm{d} \Psi} g y=\frac{\mathrm{d}(\rho B)}{\mathrm{d} \Psi} .
$$

This is the DJL equation in the Yih form [13, 2]. For the ZMV-flows, Eqs. (11) and (13) give

$$
\rho_{0} \nabla^{2} \Psi+\frac{\mathrm{d} \rho}{\mathrm{d} \Psi}\left[g y-B_{\mathrm{s}}+\frac{\psi(\Psi)-\psi_{\mathrm{s}}}{c / g}\right]=0 .
$$

Yih equation (13) is of special interest when both derivatives $\mathrm{d} \rho / \mathrm{d} \Psi$ and $\mathrm{d}(\rho B) / \mathrm{d} \Psi$ are linear functions of $\Psi$. Several solutions of this kind have been investigated by Yih (see Chap. 3 in the book [2]). It can be easily seen that the peculiar exact linear forms of the general Eq. (13) are not peculiar cases of the specific Eq. (14) because $\mathrm{d} \rho / \mathrm{d} \Psi$ and $\mathrm{d}(\rho B) / \mathrm{d} \Psi$ cannot be linear functions of $\Psi$ simultaneously for the ZMV-flows. Moreover, for the special types of the dependence $\rho(\psi)$ considered below, Yih equation (13) does not bring any simplification and therefore, it is not considered here.

(iii) Brown and Christie [11] have noticed that, for solitary waves with a uniform upstream current, Eq. (12) can be derived from the Hamilton principle. For the considered here ZMVflows, Eq. (12) also follows from that principle with the following Lagrangian density:

$$
\mathscr{L} \equiv \rho(\psi)\left[\frac{(\nabla \psi)^{2}}{2}-g y+B_{\mathrm{s}}-\frac{\psi-\psi_{\mathrm{s}}}{c / g}\right]+\frac{g}{c} \int_{\psi_{\mathrm{s}}}^{\psi} \rho(\varphi) \mathrm{d} \varphi .
$$

And the functional to be minimized is:

$$
J(\psi)=\iint_{-h}^{\eta} \mathscr{L} \mathrm{d} y \mathrm{~d} x
$$

This can be easily verified by the direct calculation.

\section{Linearly related density and stream-function}

Here we investigate steady wave motion in a stratified fluid assuming that $\rho$ linearly depends of $\psi$, whereas the dependence of unperturbed density on depth, $\rho(y)$, may be arbitrary, in general. In what follows we do not assume that the density variation with depth is small and do not use Boussinesq approximation which is traditionally exploited in the physical oceanography (see, for instance, $[1,2,3]$ ).

Without loss of generality, the linear dependence $\rho(\psi)$ can be taken in the form:

$$
\rho(\psi)=\rho_{\mathrm{s}} \psi / \psi_{\mathrm{s}},
$$

where $\rho_{\mathrm{s}}>0$ is a constant representing fluid density at the free surface. Note that this choice of linear dependence is rather general as any possible additive constant $\rho_{0}$ can be absorbed into the redefined stream function by the simple gauge transformation $\psi=\psi^{\star}-\psi_{\mathrm{s}} \rho_{0} / \rho_{\mathrm{s}}$ 
which does not affect the velocity field. In other words, the stratification (15) defines $\psi_{\mathrm{s}}$ univocally.

In accordance with Eq. (15), the constant density at the bottom is $\rho_{\mathrm{b}}=\rho_{\mathrm{s}} \psi_{\mathrm{b}} / \psi_{\mathrm{s}}=$ $\rho_{\mathrm{s}}\left(1+c h / \psi_{\mathrm{s}}\right)$, where we put $\psi_{\mathrm{b}}=\psi_{\mathrm{s}}+c h$. In this paper we consider only the statically stable case when the fluid density increases with the depth (stable stratification). This implies that $\rho_{\mathrm{b}}>\rho_{\mathrm{s}}$ and $\psi_{\mathrm{s}} / c>0$.

A fluid stratification is commonly characterized by the local buoyancy, alias BruntVäisälä, frequency $[1,2,3]$ :

$$
N_{\mathrm{L}} \equiv\left(-\frac{g}{\rho} \frac{\mathrm{d} \rho}{\mathrm{d} y}\right)^{\frac{1}{2}},
$$

where $\rho$ is unperturbed density. $N_{\mathrm{L}}$ is not constant for linearly related stratification and stream function, in general, but depends of $y$. In addition to $N_{\mathrm{L}}$, we define also a global Brunt-Väisälä frequency which is used sometimes in fluid mechanics too:

$$
N_{\mathrm{G}} \equiv\left(-\frac{g}{\rho_{\mathrm{s}}} \frac{\rho_{\mathrm{s}}-\rho_{\mathrm{b}}}{h}\right)^{\frac{1}{2}}=\left(\frac{g c}{\psi_{\mathrm{s}}}\right)^{\frac{1}{2}} .
$$

This quantity characterizes the global buoyant property of the fluid, rather than the characteristics of internal waves, whereas the local parameter $N_{\mathrm{L}}$ directly determines the dispersion properties of internal waves and their vertical structure. This implies that the parameter $\psi_{\mathrm{s}} / c$ does not depend on the wave characteristics such as wave amplitude, velocity, frequency, wavelength, etc. Hence, $\psi_{\mathrm{s}}$ must be proportional to $c$ if $N_{\mathrm{G}}$ is fixed.

In order to characterize the flow regime, we introduce some more dimensionless parameters - the Froude number Fr, global density variation $\varrho$ and densimetric Froude number Fd:

$$
\begin{aligned}
& \mathrm{Fr}^{2} \equiv \frac{c^{2}}{g h}, \quad \varrho^{2} \equiv \frac{\rho_{\mathrm{b}}-\rho_{\mathrm{s}}}{\rho_{\mathrm{s}}} \\
& \mathrm{Fd}^{2} \equiv \frac{c^{2}}{g h} \frac{\rho_{\mathrm{b}}-\rho_{\mathrm{s}}}{\rho_{\mathrm{s}}}=\left(\frac{c N_{\mathrm{G}}}{g}\right)^{2}=\varrho^{2} \mathrm{Fr}^{2} .
\end{aligned}
$$

Then, we have the relationships

$$
\frac{c^{3}}{g \psi_{\mathrm{s}}}=\mathrm{Fd}^{2}, \quad \frac{c^{3}}{g \psi_{\mathrm{b}}}=\frac{\mathrm{Fd}^{2}}{1+\varrho^{2}},
$$

which will be used later on in the subsequent sections.

Finally, for the linearly related $\rho$ and $\psi$, as per Eq. (15), and the ZMV-flows, the DJL equation (12) takes the form

$$
\psi \nabla^{2} \psi+\frac{1}{2}(\nabla \psi)^{2}+(g / c) \psi=B_{\mathrm{s}}+(g / c) \psi_{\mathrm{s}}-g y
$$

This is the governing equation which we will study first in the next section for the limiting case of infinitesimal waves. 


\section{Waves of infinitesimal amplitude}

Consider first stationary periodic waves of infinitesimal amplitude and the period $2 \pi / k$ in the background homogeneous flow. This case corresponds to the linear approximation, when the perturbation of the mean flow can be presented in the form of a harmonic wave:

$$
\psi \approx \psi_{\mathrm{s}}-c y+c f(y) a \cos (k x), \quad \eta \approx a \cos (k x),
$$

where $k a \ll 1$, and $f(y)$ is a function which must be determined. As follows from this equation, without wave perturbation, i.e., when $a=0$, the stream function linearly depends on the depth. The same is true for the density $\rho(y)$ thankful to Eq. (15).

To satisfy the impermeability boundary conditions at the surface and bottom we have to impose the following conditions on the function $f(y)$ :

$$
f(0)=1, \quad f(-h)=0 .
$$

At the free surface, the definition of the Bernoulli constant (8) yields

$$
B_{\mathrm{s}}=\frac{1}{2} c^{2}+\mathcal{O}\left(a^{2}\right),
$$

whereas the isobarity of the free surface (the condition of a constant pressure) gives in turn from Eq. (3)

$$
a \cos (k x)\left[g-c^{2} f^{\prime}(0)\right]+\mathcal{O}\left(a^{2}\right)=0,
$$

where $f^{\prime}=\mathrm{d} f / \mathrm{d} y$. Thus, neglecting the terms of the order of $a^{2}$ (and higher) we obtain in the linear approximation:

$$
c^{2}=g / f^{\prime}(0)
$$

Now, when all boundary conditions were addressed and satisfied, determine function $f(y)$; it can be found from the solution of the DJL equation (20). By substitution of the trial solution (21) into Eq. (20), one finds that to the first-order approximation in $a$, function $f(y)$ obeys the second-order linear ODE:

$$
\left[\left(\psi_{\mathrm{s}}-c y\right) f^{\prime}\right]^{\prime}+\left[g / c-k^{2}\left(\psi_{\mathrm{s}}-c y\right)\right] f=0
$$

This equation formally has a singularity at the point $y=\psi_{\mathrm{s}} / c>0$, but in the domain occupied by the fluid with the surface waves of infinitesimal amplitude, $y \leqslant 0$, therefore the singularity does not affect the solution as it is above the free surface.

Introduction of new variables

$$
z \equiv 2 k\left(\psi_{s} / c-y\right)=2 k\left(h / \varrho^{2}-y\right) \text { and } F(z) \equiv f(y) \mathrm{e}^{-k y},
$$

allows us to rewrite Eq. (26) in the standard form determining the confluent hypergeometric function [14]:

$$
z F^{\prime \prime}+(1-z) F^{\prime}-\alpha F=0
$$

where

$$
\alpha \equiv \frac{1}{2}\left(1-\frac{g}{k c^{2}}\right)=\frac{1}{2}\left(1-\frac{1}{k h \mathrm{Fr}^{2}}\right)=\frac{1}{2}\left(1-\frac{\varrho^{2}}{k h \mathrm{Fd}^{2}}\right)
$$


Introducing also the following parameters

$$
z_{\mathrm{s}} \equiv \frac{2 k \psi_{\mathrm{s}}}{c}=\frac{2 g k}{N_{\mathrm{G}}^{2}}=\frac{2 k h}{\varrho^{2}}, \quad z_{\mathrm{b}} \equiv \frac{2 k \psi_{\mathrm{b}}}{c}=\left(1+\varrho^{2}\right) z_{\mathrm{s}},
$$

we can present the boundary conditions (22) and (25) in the form:

$$
F\left(z_{\mathrm{s}}\right)=1, \quad F\left(z_{\mathrm{b}}\right)=0, \quad F^{\prime}\left(z_{\mathrm{s}}\right)=\alpha .
$$

Equation (28) together with the boundary conditions (31) forms the eigenvalue problem. Eigen solutions of Eq. (28) may be expressed in terms of special Kummer functions alias the confluent hypergeometric functions (see, e.g., [14] §13):

$$
F(z)=\frac{\mathrm{M}\left(\alpha, 1 ; z_{\mathrm{b}}\right) \mathrm{U}(\alpha, 1 ; z)-\mathrm{U}\left(\alpha, 1 ; z_{\mathrm{b}}\right) \mathrm{M}(\alpha, 1 ; z)}{\mathrm{M}\left(\alpha, 1 ; z_{\mathrm{b}}\right) \mathrm{U}\left(\alpha, 1 ; z_{\mathrm{s}}\right)-\mathrm{U}\left(\alpha, 1 ; z_{\mathrm{b}}\right) \mathrm{M}\left(\alpha, 1 ; z_{\mathrm{s}}\right)}
$$

where $\mathrm{M}$ and $\mathrm{U}$ are the Kummer functions of the first and second kind, respectively. The solution (32) fulfills the two first boundary conditions (31). The third boundary condition (31) gives the following dispersion relation

$$
\left[1+\frac{\mathrm{M}\left(\alpha, 1 ; z_{\mathrm{b}}\right) \mathrm{U}\left(\alpha+1,2 ; z_{\mathrm{s}}\right)+\mathrm{U}\left(\alpha, 1 ; z_{\mathrm{b}}\right) \mathrm{M}\left(\alpha+1,2 ; z_{\mathrm{s}}\right)}{\mathrm{M}\left(\alpha, 1 ; z_{\mathrm{b}}\right) \mathrm{U}\left(\alpha, 1 ; z_{\mathrm{s}}\right)-\mathrm{U}\left(\alpha, 1 ; z_{\mathrm{b}}\right) \mathrm{M}\left(\alpha, 1 ; z_{\mathrm{s}}\right)}\right] \alpha=0 .
$$

Introducing the dimensionless horizontal coordinate $X \equiv k x$ and stream function $\Psi \equiv$ $k \psi / c$, present solution (21) with the eigenfunction (32) in the dimensionless form

$$
\Psi(X, z)=\frac{z}{2}+\epsilon \cos (X) \exp \left(\frac{z_{\mathrm{s}}-z}{2}\right) F(z),
$$

where $\epsilon \equiv k a$. This solution contains four dimensionless parameters: $\epsilon, \alpha, z_{\mathrm{s}}$ and $z_{\mathrm{b}}$, which are directly related with the physical parameters such as the densimetric Froude number $\mathrm{Fd}$ [see Eq. (18)] and the dimensionless wave number

$$
K \equiv \frac{k c^{2}}{g}=k h \mathrm{Fr}^{2}=\frac{1}{1-2 \alpha} .
$$

Instead of analyzing this solution at once, it is enlightening and simpler to start with the limiting cases of shallow and deep fluids first.

Remark: An alternative case can be considered separately when the rigid lid boundary condition is used instead of the free-surface condition. Such condition is frequently used in physical oceanography to filter out the surface mode and focus on the internal modes only (see, e.g., $[1,2,3]$ ). In such case, stationary periodic wave of infinitesimal amplitude and period $2 \pi / k$ in the homogeneous background flow can be presented in the following form:

$$
\psi \approx c(h+y)+c f(y) a \cos (k x)
$$

with the surface value of the stream function, $\psi(0) \equiv \psi_{\mathrm{s}}=c h$. The zero boundary conditions are used at the surface and bottom:

$$
f(0)=0, \quad f(-h)=0 .
$$

The Bernoulli integral is not applicable in the rigid-lid case, therefore Eq. (25) is no longer valid. Instead of that one can use the condition of eigenfunction normalization $|f(y)|_{\max }=1$. This condition jointly with the conditions (36) and Eq. (26) forms a boundary-value problem which completely defines the structure of internal modes. 


\section{$5 \quad$ Waves in the shallow water limit}

Let us consider the shallow-water limit $(k h \ll 1)$, keeping $h$ constant and turning the wavelength to infinity $\left(k \rightarrow 0^{+}\right)$. In this case, the parameter $\alpha(29)$ goes to minus infinity, and the Kummer functions reduce to the Bessel functions (Appendix B). Then, the dispersion relation (33) becomes

$$
\begin{aligned}
& 0=\mathrm{J}_{0}\left(\frac{2 \sqrt{1+\varrho^{2}}}{\varrho \mathrm{Fr}}\right) \mathrm{Y}_{0}\left(\frac{2}{\varrho \mathrm{Fr}}\right)-\mathrm{Y}_{0}\left(\frac{2 \sqrt{1+\varrho^{2}}}{\varrho \mathrm{Fr}}\right) \mathrm{J}_{0}\left(\frac{2}{\varrho \mathrm{Fr}}\right)- \\
& \varrho \mathrm{Fr}\left[\mathrm{J}_{0}\left(\frac{2 \sqrt{1+\varrho^{2}}}{\varrho \mathrm{Fr}}\right) \mathrm{Y}_{1}\left(\frac{2}{\varrho \mathrm{Fr}}\right)-\mathrm{Y}_{0}\left(\frac{2 \sqrt{1+\varrho^{2}}}{\varrho \mathrm{Fr}}\right) \mathrm{J}_{1}\left(\frac{2}{\varrho \mathrm{Fr}}\right)\right] .
\end{aligned}
$$

where $J_{n}$ and $\mathrm{Y}_{n}$ are, respectively, the Bessel and Neumann functions of the order $n$ [14]. Note that it is rather cumbersome to derive this limiting expression from the arbitrary depth solution (32)-(33); it is much simpler to derive it directly from Eq. (26) letting $k=0$. For the given stratification $\varrho>0$, this dispersion relation has an infinite number of roots, which correspond to surface wave and set of internal wave modes. Each root defines a critical Froude number, i.e., the maximal normalized speed of infinitely long waves.

For the weak stratification $(\varrho \ll 1)$, Eq. $(37)$ yields

$$
\mathrm{Fr}^{2}-1+\left(\frac{1}{2}-\frac{1}{3} \mathrm{Fr}^{-2}\right) \varrho^{2}+\mathcal{O}\left(\varrho^{4}\right)=0
$$

In particular, for the homogeneous fluid $(\varrho=0)$, Eq. (37) defines the critical Froude number of the zeroth mode $\mathrm{Fr}=\mathrm{Fr}_{\text {crit }}^{(0)} \equiv 1$ (i.e., $c^{2}=g h$, as expected for shallow-water waves in the linear approximation). Thus, the zeroth mode is nothing but small-amplitude infinitely long surface wave. The zeroth mode is called the surface mode since it exists even in the absence of stratification.

When $\varrho>0$, in addition to the zeroth-mode root, Eq. (37) possesses an infinite number of real positive roots. These roots exist only in the presence of stratification; they are called the internal modes. Graphical solution of Eq. (37) in terms of the critical Froude numbers $\mathrm{Fr}^{(n)}$ is shown in Fig. 2 for $\varrho=1$. All roots corresponding to internal modes with $n \geqslant 1$ are close to zero if $\varrho \ll 1$, while the surface mode is close to 1 in this limit. This can be seen from the asymptotic expansion of Eq. (37) which, after some algebra, yields for $\mathrm{Fr} \rightarrow 0$ :

$$
\varrho \operatorname{Fr} \sim \tan \left(\frac{\sqrt{1+\varrho^{2}}-1}{\varrho \operatorname{Fr} / 2}\right) .
$$

The critical Froude numbers are designated as $\mathrm{Fr}_{\text {crit }}^{(n)}(n=0,1,2, \cdots)$ and sorted in the decreasing order of magnitude (so that $\mathrm{Fr}^{(n)} \rightarrow 0$ as $n \rightarrow \infty$ ). The corresponding critical phase velocities and eigenfunctions are then designated as $c_{\mathrm{sf}}^{(n)}$ and $f_{\mathrm{sf}}^{(n)}$, respectively, where the subscript 'sf' stands for the 'shallow fluid'.

The critical Froude numbers $\mathrm{F}_{\mathrm{crit}}^{(n)}$ depend of the stratification parameter $\varrho$. As $\varrho$ increases, the zeroth-mode critical Froude number $\mathrm{F}_{\text {crit }}^{(0)}$ monotonically decreases, while other critical 
Froude numbers monotonically increase (Fig. 3). However, there is no intersections between the roots even when $\varrho \rightarrow \infty$. The limiting values of the roots are $\operatorname{Fr}_{\text {crit }}^{(0)}(\varrho=\infty) \approx 0.832$, $\operatorname{Fr}_{\text {crit }}^{(1)}(\varrho=\infty) \approx 0.362, \operatorname{Fr}_{\text {crit }}^{(2)}(\varrho=\infty) \approx 0.231, \operatorname{Fr}_{\text {crit }}^{(3)}(\varrho=\infty) \approx 0.169$, etc. They are shown in Fig. 3 by horizontal dashed lines. Thus, there is a finite gap between the possible velocities of the surface and internal modes, e.g., $\operatorname{Fr}_{\text {crit }}^{(0)}(\varrho=\infty)-\operatorname{Fr}_{\text {crit }}^{(1)}(\varrho=\infty) \approx 0.47$.

In the limit $\varrho=0$, only the zeroth-mode exists, the corresponding velocity field turns into the uniform current, and the eigenfunction $f_{\mathrm{sf}}^{(0)}(y)$ becomes a linear function of $y$ :

$$
f_{\mathrm{sf}}^{(0)}(y)=1+y / h+\mathcal{O}\left(\varrho^{2}\right) .
$$

If $\varrho>0$, the infinite set of internal modes do exist. The current induced by each mode is described by the following eigenfunctions, which follows from Eqs. (27) and (32) in the limit of $k h \rightarrow 0$ :

$$
f_{\mathrm{sf}}^{(n)}(y)=\frac{\mathrm{Y}_{0}\left(G_{n} \sqrt{1-\varrho^{2} \frac{y}{h}}\right)-\mathrm{R}_{0}\left(G_{n} \sqrt{1+\varrho^{2}}\right) \mathrm{J}_{0}\left(G_{n} \sqrt{1-\varrho^{2} \frac{y}{h}}\right)}{\mathrm{Y}_{0}\left(G_{n}\right)-\mathrm{R}_{0}\left(G_{n} \sqrt{1+\varrho^{2}}\right) \mathrm{J}_{0}\left(G_{n}\right)},
$$

where $G_{n}=2 / \varrho \mathrm{Fr}_{\text {crit }}^{(n)}$, and function $\mathrm{R}_{0}(x)=\mathrm{Y}_{0}(x) / \mathrm{J}_{0}(x)$.

The structures of the first three modes are presented in Fig. 4 for the case when $\varrho=1$. As one can see from this figure, the modes structure is qualitatively similar to the structure of linear modes in the exponentially stratified fluid, when the Brunt-Väisälä frequency $N_{\mathrm{L}}=$ const [3]. Notice also that our definition of eigenfunctions $f_{\mathrm{sf}}^{(n)}(y)$, as per Eq. (21), is such that each mode has the same velocity $c$; this results in the boundary conditions (22) and (25). Hence, all eigenfunctions turn to unity at the fluid surface, whereas their maxima and minima attained in the bulk of the fluid may be much greater in absolute value than one, as it is illustrated in Fig. 4.

In the asymptotic limit $\mathrm{Fr}_{\text {crit }}^{(n)} \rightarrow 0$, the expansion of Eq. (38) yields:

$$
f_{\mathrm{sf}}^{(n)}(y) \sim \frac{\sin \left[\left(2 / \varrho \mathrm{Fr}_{\text {crit }}^{(n)}\right)\left(\sqrt{1+\varrho^{2}}-\sqrt{1-\varrho^{2} y / h}\right)\right]}{\sqrt[4]{1-\varrho^{2} y / h} \sin \left[\left(2 / \varrho \mathrm{Fr}_{\text {crit }}^{(n)}\right)\left(\sqrt{1+\varrho^{2}}-1\right)\right]} .
$$

It can be readily seen from this formula that the number of nodes of the eigenfunction $f_{\mathrm{sf}}^{(n)}(y)$ increases with the mode number $n$ as $\mathrm{Fr}_{\text {crit }}^{(n)}$ tends to zero. The behavior of the eigenvalues $\mathrm{F}_{\mathrm{crit}}^{(n)}$ and eigenfunctions $f_{\mathrm{sf}}^{(n)}(y)$ is in accordance with the general Sturm oscillation theorem $[15]$.

\section{Waves in the deep-water limit}

In the deep-water limit (i.e., when $h \rightarrow \infty$ and hence, $\left\{\psi_{\mathrm{b}}, \rho_{\mathrm{b}}, z_{\mathrm{b}}\right\} \rightarrow \infty$ ) solution (32) and the dispersion relation (33) become

$$
F(z)=\frac{\mathrm{U}(\alpha, 1 ; z)}{\mathrm{U}\left(\alpha, 1 ; z_{\mathrm{s}}\right)}, \quad\left[1+\frac{\mathrm{U}\left(\alpha+1,2 ; z_{\mathrm{s}}\right)}{\mathrm{U}\left(\alpha, 1 ; z_{\mathrm{s}}\right)}\right] \alpha=0
$$


with

$$
\alpha=\frac{1}{2}-\frac{1}{2} K^{-1}, \quad z_{\mathrm{s}}=2 \mathrm{KFd}^{-2} .
$$

Notice that in the deep-water limit, the Froude number Fr as defined above is zero, but the densimetric Froude number Fd may be finite, in general. Similar to the shallow-water case, for the fixed parameter $\mathrm{Fd}>0$, the dispersion relation has the infinite number of roots (Fig. 5), whereas there is only one root if $\mathrm{Fd}=0$. Below we explain this fact.

In the limiting case of a homogeneous fluid, i.e. when $\varrho=0$, and $\left\{z, z_{\mathrm{s}}\right\}=+\infty$, whereas $z / z_{\mathrm{s}}=1$, solution (39) for the eigenfunction becomes trivial ${ }^{1}$ :

$$
F(z)=1, \quad \alpha=0 .
$$

According to Eqs. (27) and (29), this yields $f(y)=\exp (k y)$ and $c^{2}=g / k$ in agreement with the linear theory of surface gravity waves in the deep homogeneous fluid. This solution, corresponding to the zeroth mode, is characterized by the dimensionless wave number $K_{\mathrm{df}}^{(0)} \equiv$ $k c^{2} / g=1$ (subscript 'df' stand for the 'deep fluid').

For the heterogeneous fluid $(\varrho>0)$, the infinite number of other roots of the dispersion relation (39) appears in addition to the zeroth mode, $\alpha=0$. These roots are solutions of the equation

$$
\mathrm{U}\left(\alpha+1,2 ; z_{\mathrm{s}}\right)+\mathrm{U}\left(\alpha, 1 ; z_{\mathrm{s}}\right)=0 .
$$

The corresponding eigenmodes are numbered in the decreasing order of magnitude of the dimensionless wave number $K$, i.e. $K_{\mathrm{df}}^{(0)}>K_{\mathrm{df}}^{(1)}>K_{\mathrm{df}}^{(2)}>\cdots$ (see Fig. 5). Each root $K_{\mathrm{df}}^{(n)}$ increases when the stratification becomes stronger, i.e. when $\varrho$ increases.

In contrast to the zeroth mode, the eigenfunctions $f^{(n)}(y)$ do not vanish monotonically when $y \rightarrow-\infty$ (see Fig. 6). The eigenfunction $f^{(n)}(y)$ of the $n$ th-mode has $n$ local extrema for $0>y>-\infty$. The behavior of the eigenvalues and eigenfunctions is again in the complete accordance with the Sturm oscillation theorem [15].

\section{The general case of a finite-depth fluid}

In the general case of the finite-depth fluid, the dispersion relation (33), similar to two previous cases of shallow and deep water, has an infinite number of roots if $\varrho \neq 0$, and only one root if $\varrho=0$. However, in contrast to the previous case, in general, $\alpha=0$ is not a root of Eq. (33) if $h<\infty$, because the expression in the square brackets of Eq. (33) goes to infinity, when $\alpha \rightarrow 0$. In the result of this the first term of the Taylor expansion of Eq. (33) around $\alpha=0$ is (for details see Appendix B):

$$
\frac{\exp \left(z_{\mathrm{s}}\right)}{z_{\mathrm{s}}} \frac{1}{\operatorname{chi}\left(z_{\mathrm{b}}\right)-\operatorname{chi}\left(z_{\mathrm{s}}\right)+\operatorname{shi}\left(z_{\mathrm{b}}\right)-\operatorname{shi}\left(z_{\mathrm{s}}\right)}+\mathcal{O}(\alpha)=0
$$

where $\operatorname{chi}(x)$ and $\operatorname{shi}(x)$ are the hyperbolic cosine and sine integral functions, respectively [14].

\footnotetext{
${ }^{1}$ Note that the expression in the square brackets of the dispersion relation (39) remains bounded when $\alpha \rightarrow 0$.
} 
In the limiting case of a homogeneous fluid $(\varrho=0)$, the dispersion relation (33) reduces after some (rather cumbersome) algebra to the usual dispersion relation for surface water waves in the fluid of finite depth $[1,3]$ :

$$
\frac{c^{2}}{g h}=\frac{\tanh (k h)}{k h}
$$

In the case $\varrho \neq 0$, no further simplification of Eq. (33) were found, and the roots of this equation, including the zero-mode root, can be found only numerically.

\section{Solitary waves}

For solitary waves, the flow represents a uniform current in the far field. If we consider solitary waves decaying exponentially ${ }^{2}$, than it is natural to seek for a solution having the following asymptotic behavior when $x \rightarrow \pm \infty$ :

$$
\psi \sim \psi_{\mathrm{s}}-c y+a c \exp (-\kappa|x|) f(y), \quad \eta \sim a \exp (-\kappa|x|),
$$

where $\kappa \geqslant 0$ is a parameter characterizing the spatial decay of the solitary wave. Substitution of this asymptotic expression for $\psi$ into governing equation (20) gives a straightforward derivation of the wave velocity $c$. However, to obtain the result, it is even simpler to apply the transformation $k \mapsto \mathrm{i} \kappa$ (where $\mathrm{i}^{2}=-1$ ) to the dispersion relation (33), which was formally derived in the linear approximation consistent with the far-field asymptotic of the soliton tail. In the result, we obtain:

$$
\mathrm{U}\left(\hat{\alpha}, 1 ; \mathrm{i} \hat{z}_{\mathrm{s}}\right)+\mathrm{U}\left(\hat{\alpha}+1,2 ; \mathrm{i} \hat{z}_{\mathrm{s}}\right)+\frac{\mathrm{U}\left(\hat{\alpha}, 1 ; \mathrm{i} \hat{z}_{\mathrm{b}}\right)}{\mathrm{M}\left(\hat{\alpha}, 1 ; \mathrm{i} \hat{z}_{\mathrm{b}}\right)}\left[\mathrm{M}\left(\hat{\alpha}+1,2 ; \mathrm{i} \hat{z}_{\mathrm{s}}\right)-\mathrm{M}\left(\hat{\alpha}, 1 ; \mathrm{i} \hat{z}_{\mathrm{s}}\right)\right]=0,
$$

with

$$
\hat{\alpha}=\frac{1}{2}\left(1+\frac{\mathrm{i} g}{\kappa c^{2}}\right), \quad \hat{z}_{\mathrm{s}}=\frac{2 \kappa h}{\varrho^{2}}, \quad \hat{z}_{\mathrm{b}}=\left(1+\varrho^{2}\right) \hat{z}_{\mathrm{s}} .
$$

In the limiting case of a homogeneous fluid $(\varrho=0)$, the dispersion relation (45) yields

$$
\frac{c^{2}}{g h}=\frac{\tan (\kappa h)}{\kappa h} \text {. }
$$

This formula was derived for the first time by McCowan [16]. Stokes [17] noticed that Eq. (47) is actually the exact relation for a solitary wave of arbitrary amplitude, unlike Eq. (43), which is valid for sinusoidal waves of infinitesimal amplitude. Similarly, the dispersion relation (45) is exact for solitary waves of arbitrary amplitude, whereas Eq. (33) is just an approximation for sinusoidal waves of infinitesimal amplitude.

In the limit of $\kappa h \rightarrow 0$, it follows from Eq. (47):

$$
c \approx \sqrt{g h}\left(1+\frac{\kappa h}{3}\right) .
$$

\footnotetext{
${ }^{2}$ Algebraic solitary waves, whose asymptotic decay is of a power type $\sim x^{-p}$, where $p>0$ is some constant, may be also considered, but in a separate representation.
} 
This formula can be compared with the known dependence of soliton velocity on depth which follows from the Boussinesq or Korteweg-de Vries theory in the shallow-water limit, $\kappa h \rightarrow 0$, (see, e.g., [18]):

$$
c \approx \sqrt{g h}\left(1+\frac{\eta_{0}}{2 h}\right)
$$

where $\eta_{0}$ is the amplitude of a surface soliton. By comparison of Eqs. (48) and (49), we can identify (at least in this shallow-water limit) $\kappa=3 \eta_{0} / 2 h^{2}$. For large depths and amplitudes this relation between $\kappa$ and $\eta_{0}$ is not valid, nevertheless substituting it formally into Eq. (47), we may roughly estimate the maximal possible amplitude of the surface soliton when its velocity turns to infinity: $\left(\eta_{0}\right)_{\max } \approx \pi h / 3 \approx h$, whereas more precise value is $\left(\eta_{0}\right)_{\max } \approx 0.83 h$ (see [19] and references therein).

\section{Other cases of density dependences on the stream function}

As has been shown above, when the fluid density is linearly related to the stream function, the linearized problem for infinitesimal waves can be solved in the closed analytical form. This gives an useful insight to the problem of water waves in stratified fluid and helps in the more advanced analytical and numerical investigations. However, in some more complicated cases when the density and stream function are related by certain nonlinear functions, the problem of analytical description of water waves is still tractable to analysis. Below we consider some of such cases.

\subsection{The generic density function}

Let us consider a general dependence between $\rho$ and $\psi$ in the form:

$$
\rho(\psi)=\rho_{\mathrm{s}} Q\left(\psi / \psi_{\mathrm{s}}\right),
$$

where $\rho_{\mathrm{s}}>0$ is a constant representing fluid density at the free surface and $Q$ is a nondecreasing function such that $Q(1)=1$. If $\mathrm{d} \rho / \mathrm{d} \psi \neq 0$, one may impose $Q(0)=0$ or any other convenient relation providing a gauge condition for $\psi$. The constant density at the bottom is $\rho_{\mathrm{b}}=\rho_{\mathrm{s}} Q\left(1+c h / \psi_{\mathrm{s}}\right)$. For statically stable stratifications, $\rho_{\mathrm{b}}>\rho_{\mathrm{s}}$ and $\psi_{\mathrm{s}} / c>0$.

\subsection{The power density dependence}

A first example having a special interest consider the power dependence of the density on the stream function:

$$
\rho(\psi)=\rho_{\mathrm{s}}\left(\psi / \psi_{\mathrm{s}}\right)^{\beta},
$$

where $\beta$ is a constant. This relation reduces to the cases of homogeneous fluid, if $\beta=0$, and linearly related $\rho$ and $\psi$, if $\beta=1$.

The DJL equation (12) with the relationship (51) reads:

$$
\beta^{-1} \psi \nabla^{2} \psi+\frac{1}{2}(\nabla \psi)^{2}+(g / c) \psi=B_{\mathrm{S}}+(g / c) \psi_{\mathrm{s}}-g y,
$$


This equation only slightly differ from Eq. (20) due to the coefficient $\beta$ in front of the first term. For the waves of infinitesimal amplitude described by Eq. (21), equation (52) can be also solved in the closed analytical form. The corresponding equation for the eigenfunction $f(y)$ in this case is (cf. Eq. (26)):

$$
\left[\left(\psi_{\mathrm{s}}-c y\right) f^{\prime}\right]^{\prime}+(1-\beta) c f^{\prime}+\left(\beta g / c-k^{2} \psi_{\mathrm{s}}+k^{2} c y\right) f=0 .
$$

Introduction of new variables and parameters

$$
\begin{gathered}
z \equiv 2 k\left(\psi_{s} / c-y\right), \quad F(z) \equiv f(y) \mathrm{e}^{-k y}, \quad \alpha \equiv \frac{\beta}{2}\left(1-\frac{g}{k c^{2}}\right) \\
z_{\mathrm{s}} \equiv \frac{2 k \psi_{\mathrm{s}}}{c}=\frac{2 g k}{N_{\mathrm{G}}^{2}}=\frac{2 k h}{\varrho^{2}}, \quad z_{\mathrm{b}} \equiv \frac{2 k \psi_{\mathrm{b}}}{c}=\left(1+\varrho^{2}\right) z_{\mathrm{s}}
\end{gathered}
$$

allows us to rewrite Eq. (53) in the standard form determining the confluent hypergeometric function [14]:

$$
z F^{\prime \prime}+(\beta-z) F^{\prime}-\alpha F=0
$$

The latter equation augmented by the boundary conditions [cf. Eq. (31)]:

$$
F\left(z_{\mathrm{s}}\right)=1, \quad F\left(z_{\mathrm{b}}\right)=0, \quad F^{\prime}\left(z_{\mathrm{s}}\right)=\alpha / \beta .
$$

forms the eigenvalue problem. Solution of Eq. (56) may be expressed again in terms of the confluent hypergeometric functions (see, e.g., $§ 13$ in the book [14]):

$$
F(z)=\frac{\mathrm{M}\left(\alpha, \beta ; z_{\mathrm{b}}\right) \mathrm{U}(\alpha, \beta ; z)-\mathrm{U}\left(\alpha, \beta ; z_{\mathrm{b}}\right) \mathrm{M}(\alpha, \beta ; z)}{\mathrm{M}\left(\alpha, \beta ; z_{\mathrm{b}}\right) \mathrm{U}\left(\alpha, \beta ; z_{\mathrm{s}}\right)-\mathrm{U}\left(\alpha, \beta ; z_{\mathrm{b}}\right) \mathrm{M}\left(\alpha, \beta ; z_{\mathrm{s}}\right)}
$$

The solution (58) fulfills two first boundary conditions (57), whereas the third boundary condition (57) yields the dispersion relation:

$$
\left[1+\frac{\beta \mathrm{M}\left(\alpha, \beta ; z_{\mathrm{b}}\right) \mathrm{U}\left(\alpha+1, \beta+1 ; z_{\mathrm{s}}\right)+\mathrm{U}\left(\alpha, \beta ; z_{\mathrm{b}}\right) \mathrm{M}\left(\alpha+1, \beta+1 ; z_{\mathrm{s}}\right)}{\mathrm{M}\left(\alpha, \beta ; z_{\mathrm{b}}\right) \mathrm{U}\left(\alpha, \beta ; z_{\mathrm{s}}\right)-\mathrm{U}\left(\alpha, \beta ; z_{\mathrm{b}}\right) \mathrm{M}\left(\alpha, \beta ; z_{\mathrm{s}}\right)}\right] \frac{\alpha}{\beta}=0 .
$$

As in the case of linearly related functions $\rho$ and $\psi$, this dispersion relation has an infinite number of real roots, if $\varrho>0$, and only one root, if $\varrho=0$. The roots with pure imaginary wave numbers $k$ correspond to solitary waves.

\subsection{The exponential dependence of the density on the stream function}

Another interesting example leading to exactly solvable model is the case when fluid density $\rho$ exponentially depends on the stream function $\psi$ :

$$
\rho(\psi)=\rho_{\mathrm{s}} \exp \left[-\gamma\left(1-\psi / \psi_{\mathrm{s}}\right)\right]
$$

where $\gamma$ is a constant (positive in the case of stable stratification). 
Let us define the analog of the Brunt-Väisälä frequency as follows [cf. Eq. (16)]:

$$
\mathcal{N}(\psi) \equiv\left(\frac{g c}{\rho} \frac{\mathrm{d} \rho}{\mathrm{d} \psi}\right)^{\frac{1}{2}} .
$$

This parameter is constant in the case of exponential dependence of $\rho(\psi)(60): \mathcal{N}=$ $\sqrt{\gamma g c / \psi_{\mathrm{s}}}$. In this particular case, the DJL equation (12) reads:

$$
\nabla^{2} \psi+\frac{\mathcal{N}^{2}}{g c}\left[\frac{1}{2}(\nabla \psi)^{2}-B_{\mathrm{s}}+(g / c)\left(\psi-\psi_{\mathrm{s}}\right)+g y\right]=0 .
$$

For the waves of infinitesimal amplitude as per Eq. (21) taking into account Eq. (23) one obtains the corresponding equation for the eigenfunction $f(y)$ :

$$
f^{\prime \prime}-\frac{\mathcal{N}^{2}}{g} f^{\prime}+k^{2}\left[\left(\frac{\mathcal{N}}{c k}\right)^{2}-1\right] f=0 .
$$

This constant coefficients second-order ODE can be readily solved. The solution subject to the boundary conditions $(22)$ is:

$$
f(y)=\frac{\sin [\delta(y+h)]}{\sin (\delta h)} \exp \left(\frac{\mathcal{N}^{2} y}{2 g}\right),
$$

provided that $\delta \equiv k \sqrt{\mathcal{N}^{2} /(c k)^{2}-1-\left(\mathcal{N}^{2} / 2 g k\right)^{2}}$ is real. The dynamic boundary condition at the water surface $(25)$ yields the dispersion relation

$$
\delta h=\frac{g h}{c^{2}}\left[1-\frac{1}{2}\left(\frac{c \mathcal{N}}{g}\right)^{2}\right] \tan (\delta h) .
$$

This equation has the zero-mode root at the point $\delta h=0$,

$$
c_{0}^{2}=\frac{g h}{1+\mathcal{N}^{2} h /(2 g)},
$$

and numerous internal modes, whose roots are equal approximately to

$$
c_{n}^{2} \approx \frac{\mathcal{N}^{2}}{k^{2}+\pi^{2}(2 n+1)^{2} /\left(4 h^{2}\right)+\mathcal{N}^{4} /\left(4 g^{2}\right)},
$$

where $n=1,2,3 \ldots$, and the higher the mode number $n$, the more accurate formula (67) is. Notice also the well-known fact that the wave speed decreases with the mode number for the fixed value of $k$. These results agree with the results by Yanowitch [20] who has proven that the greatest speed of water waves which can be attained in stratified fluid with piecewise smooth density profile is $(g h)^{1 / 2}$.

If, however, the parameter $\delta$ (see above) is pure imaginary, so that $\delta=i \kappa$ where $\kappa$ is real, then the solution of Eq. (62) subject to the boundary conditions (22) is

$$
f(y)=\frac{\sinh [\kappa(y+h)]}{\sinh (\kappa h)} \exp \left(\frac{\mathcal{N}^{2} y}{2 g}\right) .
$$


And the dynamic boundary condition at the water surface (25) yields the dispersion relation

$$
\kappa h=\frac{g h}{c^{2}}\left[1-\frac{1}{2}\left(\frac{c \mathcal{N}}{g}\right)^{2}\right] \tanh (\kappa h) .
$$

This equation has only one solution given by Eq. (66) for the zero mode at the point $\kappa h=0$.

\subsection{The power-exponential dependence}

All above considered cases can be presented through the following combination of power and exponential dependence of the density on the stream function:

$$
\rho(\psi)=\rho_{\mathrm{s}}\left(\psi / \psi_{\mathrm{s}}\right)^{\beta} \exp \left[-\gamma\left(1-\psi / \psi_{\mathrm{s}}\right)\right]
$$

where $\beta$ and $\gamma$ are constants. If $\gamma=0$, this dependence naturally reduces to the case of the power-type stratification, Eq. (51), whereas if $\beta=0$, the dependence reduces to the exponential stratification, Eq. (60).

For the peculiar stratification (70), the DJL equation (12) becomes

$$
\psi \nabla^{2} \psi+\left(\beta+\gamma \psi / \psi_{\mathrm{s}}\right)\left[\frac{1}{2}(\nabla \psi)^{2}-B_{\mathrm{s}}+(g / c)\left(\psi-\psi_{\mathrm{s}}\right)+g y\right]=0 .
$$

Then, for the waves of infinitesimal amplitude as per Eq. (21), the following equations follows from Eq. (71)

$$
f^{\prime \prime}-c\left(\frac{\beta}{\psi_{s}-c y}+\frac{\gamma}{\psi_{\mathrm{s}}}\right) f^{\prime}+\frac{g}{c}\left(\frac{\beta}{\psi_{s}-c y}+\frac{\gamma}{\psi_{\mathrm{s}}}-\frac{c k^{2}}{g}\right) f=0 .
$$

With the help of new variables

$$
z \equiv 2 k \delta\left(\psi_{s} / c-y\right), \quad F(z) \equiv f(y) \mathrm{e}^{-\left(k \delta+\gamma c / 2 \psi_{\mathrm{s}}\right) y}
$$

and parameters

$$
\delta \equiv \sqrt{1-\frac{\gamma g}{k^{2} c \psi_{\mathrm{s}}}+\left(\frac{\gamma c}{2 k \psi_{\mathrm{s}}}\right)^{2}}, \quad \alpha \equiv \frac{\beta}{2}\left(1-\frac{g}{k \delta c^{2}}+\frac{c \gamma}{2 k \delta \psi_{\mathrm{s}}}\right),
$$

equation (72) can be rewritten in the standard form (56) determining the confluent hypergeometric function. The boundary conditions for the function $F(z)$ are the same as in Eqs. (57).

The solution of the boundary value problem is formally given by Eqs. (58) and (59) with different definition of the variables and parameters.

\subsection{The rational dependence of the density on the stream function}

In addition to the previously considered cases of the relationship between the fluid density and stream function, there is also the case of rational dependence between these quantities, which also leads to the tractable equation for infinitesimal waves. To derive the corresponding 
governing equation, note first that the factor $(1 / \rho)(\mathrm{d} \rho / \mathrm{d} \psi)$, appearing as the multiplier in front of the square brackets in the DJL equation (12), for the power-exponential dependence (70) considered above is:

$$
\frac{\psi_{\mathrm{s}}}{\rho} \frac{\mathrm{d} \rho}{\mathrm{d} \psi}=\frac{\beta+\gamma \psi / \psi_{\mathrm{s}}}{\psi / \psi_{\mathrm{s}}}
$$

This is a particular case of the rational function, and its generalization is an arbitrary rational function:

$$
\frac{\psi_{\mathrm{s}}}{\rho} \frac{\mathrm{d} \rho}{\mathrm{d} \psi}=\frac{R\left(\psi / \psi_{\mathrm{s}}\right)}{S\left(\psi / \psi_{\mathrm{s}}\right)} \Rightarrow \rho(\psi)=\rho_{\mathrm{s}} \exp \left[\int_{1}^{\frac{\psi}{\psi_{\mathrm{s}}}} \frac{R(\varphi)}{S(\varphi)} \mathrm{d} \varphi\right],
$$

where $R(\varphi)$ and $S(\varphi)$ are the polynomials in $\varphi$ of the degrees $r$ and $s$, respectively. With the suitable choice of these polynomials, a fairly complicated stratification can be modeled on the basis of the Padé-like approximation.

Substituting (76) into the DJL equation (12), we obtain

$$
\psi_{\mathrm{s}} \nabla^{2} \psi+\frac{R\left(\psi / \psi_{\mathrm{s}}\right)}{S\left(\psi / \psi_{\mathrm{s}}\right)}\left[\frac{1}{2}(\nabla \psi)^{2}-B_{\mathrm{s}}+(g / c)\left(\psi-\psi_{\mathrm{s}}\right)+g y\right]=0 .
$$

For the waves of infinitesimal amplitude, substitution here the ansatz (21) yields

$$
\psi_{\mathrm{s}} \bar{S}\left(f^{\prime \prime}-k^{2} f\right)-c \bar{R} f^{\prime}+\left[(g / c) \bar{R}+\left(c^{2} / 2 \psi_{\mathrm{s}}\right) \bar{R}^{\prime}\right] f=0 .
$$

where $\bar{R} \equiv R\left(1-c y / \psi_{\mathrm{s}}\right)$ and $\bar{S} \equiv S\left(1-c y / \psi_{\mathrm{s}}\right)$ are polynomials in $y$ in the small-amplitude approximation.

Equation (78) represents again a linear second-order ODE with polynomial coefficients. It can be solved in terms of generalized hypergeometric functions. Augmenting Eq. (78) by the boundary conditions (22) and (25), one obtains the boundary-value problem which allows one to find the discrete spectrum of eigenvalues and corresponding eigenfunctions.

Thus, the structure of the stationary wave field in the continuously stratified fluid can be effectively solved, at least, for the waves of infinitesimal amplitudes. In addition to that, velocities of solitary waves can be also found. Such solutions are of a practical interest allowing one to gain physical insights to the problem. They can be used also as a starting point for more advanced analytical and numerical investigations.

\section{Conclusion}

In this paper we introduced a wide class of stationary nonlinear wave motions with zero mean vorticity in density stratified fluids. The equations describing such motions were derived and analyzed. The characteristic features of wave motions with the ZMV are similar, to certain extant, to the potential waves in nonstratified fluids. For the waves of infinitesimal amplitudes the boundary-value problems can be analytically solved in many cases when the fluid density is a specific function of the stream function. Both the eigenvalues and the corresponding eigenfunctions can be found in the closed analytical form without usage of Boussinesq ap-

proximation. Examples of dispersion relations were obtained for deep and shallow water in 
the case when water density linearly depends of the stream function. Eigenmodes were also obtained both for the surface and internal modes.

It was also shown that the velocity of a finite amplitude solitary waves can be deduced in the exact form for any amplitude of the wave in density stratified fluid.

\section{Appendices}

\section{A Complements on the derivations}

With the change of independent variables $(x, y) \mapsto(x, \psi)$ and introduction of dependent variable $y=Y(x, \psi)$, we have the following transformation of derivatives

$$
\frac{\partial \bullet}{\partial x} \mapsto \frac{\partial \bullet}{\partial x}-\frac{Y_{x}}{Y_{\psi}} \frac{\partial \bullet}{\partial \psi}, \quad \frac{\partial \bullet}{\partial y} \mapsto \frac{1}{Y_{\psi}} \frac{\partial \bullet}{\partial \psi}, \quad \frac{\mathrm{D} \bullet}{\mathrm{D} t} \mapsto \frac{1}{Y_{\psi}} \frac{\partial \bullet}{\partial x},
$$

and the relationships

$$
\begin{gathered}
u \mapsto U=\frac{1}{Y_{\psi}}, \quad v \mapsto V=\frac{Y_{x}}{Y_{\psi}}, \\
\omega \mapsto \Omega=\frac{\partial}{\partial x}\left(\frac{Y_{x}}{Y_{\psi}}\right)-\frac{\partial}{\partial \psi}\left(\frac{1+Y_{x}^{2}}{2 Y_{\psi}^{2}}\right),
\end{gathered}
$$

where $Y_{x} \equiv \partial Y / \partial x$ and $Y_{\psi} \equiv \partial Y / \partial \psi$.

For $(2 \pi / k)$-periodic waves, the definition of $\omega(x, y)=\Omega(x, \psi)$ in Eq. (81) implies that the mean vorticity is

$$
\langle\Omega\rangle=-\frac{\mathrm{d}}{\mathrm{d} \psi}\left\langle\frac{1+Y_{x}^{2}}{2 Y_{\psi}^{2}}\right\rangle=-\frac{\mathrm{d}}{\mathrm{d} \psi}\left\langle\frac{U^{2}+V^{2}}{2}\right\rangle,
$$

where the averaging over $x$, denoted by angular brackets, $\langle\bullet\rangle$, is taken along streamlines, i.e. keeping $\psi$ constant. Thus, if we define the ZMV-flow with $\langle\omega\rangle=0$, the relation (82) implies that $\left\langle u^{2}+v^{2}\right\rangle$ is independent of $\psi$. Hence, the constant pressure condition at the free surface yields in turn [see Eq. (8)]:

$$
\left\langle u^{2}+v^{2}\right\rangle=\left\langle u_{\mathrm{s}}^{2}+v_{\mathrm{s}}^{2}\right\rangle=2 B_{\mathrm{s}} .
$$

In accordance with definition of the ZMV-flow, the mean horizontal velocity of such flow is a uniform current. This implies that the mean velocity between two arbitrarily chosen streamlines is constant. Considering the free surface $y=\eta(x)$ as the first streamline and $y=Y(x, \psi)$ as another arbitrarily chosen streamline, we have (see the definition (7) of the phase velocity $c)$ :

$$
c=-\frac{\int_{-\pi / k}^{\pi / k} \int_{Y}^{\eta} u \mathrm{~d} y \mathrm{~d} x}{\int_{-\pi / k}^{\pi / k} \int_{Y}^{\eta} 1 \mathrm{~d} y \mathrm{~d} x}=-\frac{\int_{-\pi / k}^{\pi / k} \int_{\psi}^{\psi_{\mathrm{s}}} 1 \mathrm{~d} \psi \mathrm{d} x}{\int_{-\pi / k}^{\pi / k} \int_{\psi}^{\psi_{\mathrm{s}}} Y_{\psi} \mathrm{d} \psi \mathrm{d} x} .
$$


From the definition of the mean surface level as per (6), it follows at once that

$$
\langle Y\rangle \equiv \frac{k}{2 \pi} \int_{-\pi / k}^{\pi / k} Y(x, \psi) \mathrm{d} x=\frac{\psi_{\mathrm{s}}-\psi}{c} .
$$

Averaging now the DJL equation over the wave period (5), we obtain

$$
\frac{\mathrm{d}(\rho B)}{\mathrm{d} \psi}=\left[B_{\mathrm{s}}+\frac{g\left(\psi_{\mathrm{s}}-\psi\right)}{c}\right] \frac{\mathrm{d} \rho}{\mathrm{d} \psi} .
$$

Then, after some elementary algebra, we deduce

$$
B(\psi)=B_{\mathrm{s}}+\frac{g\left(\psi_{\mathrm{s}}-\psi\right)}{c}+\frac{g}{\rho c} \int_{\psi_{\mathrm{s}}}^{\psi} \rho(\varphi) \mathrm{d} \varphi .
$$

This equation defines the Bernoulli 'constant' for the peculiar ZMV-flow. In the case of linear relationship between the density and stream function, i.e. $\rho=\rho_{\mathrm{s}} \psi / \psi_{\mathrm{s}}$, we have

$$
B(\psi)=B_{\mathrm{s}}-\frac{g\left(\psi_{\mathrm{s}}-\psi\right)^{2}}{2 c \psi} .
$$

Note that for a solitary wave $(k \rightarrow 0)$, the flow in the far field $(x \rightarrow \pm \infty)$ is a uniform current, i.e.

$$
u \sim-c, \quad v \sim 0, \quad \omega \sim 0, \quad Y \sim\left(\psi_{\mathrm{s}}-\psi\right) / c,
$$

and therefore the Bernoulli 'constant' is given by

$$
B(\psi)=\frac{c^{2}}{2}+\frac{g\left(\psi_{\mathrm{s}}-\psi\right)}{c}+\frac{g}{\rho c} \int_{\psi_{\mathrm{s}}}^{\psi} \rho(\varphi) \mathrm{d} \varphi .
$$

The relation (87) with $B_{\mathrm{S}}=\frac{1}{2} c^{2}$ is thus recovered. This shows that the fluid motion with the zero mean vorticity is a natural generalization of periodic waves for the case of solitary waves with the uniform upstream current.

Note also that in the case of deep fluid $(h=\infty)$ and ZMV-flow, the motion tends to a uniform current when $y \rightarrow-\infty$ and thus, $\left\langle u^{2}+v^{2}\right\rangle=c^{2}=2 B_{\mathrm{s}}$.

\section{B Kummer functions and their properties}

For the easy references, we introduce here the Kummer functions (confluent hypergeometric functions) and give few their properties used in this paper. More information can be found in special books $[21,14,22]$.

The Kummer function of the first kind $\mathrm{M}$ is defined as

$$
\mathrm{M}(\alpha, \beta ; z) \equiv \sum_{n=0}^{\infty} \frac{(\alpha)_{n}}{(\beta)_{n}} \frac{z^{n}}{n !},
$$


where $(\alpha)_{n} \equiv \alpha(\alpha+1)(\alpha+2) \cdots(\alpha+n-1)$ is the Pochhammer symbol. The function M is also sometimes denoted by ${ }_{1} \mathrm{~F}_{1}$ or $\Phi$ in the literature.

The Kummer functions of the second kind $U$ is defined as

$$
\mathrm{U}(\alpha, \beta ; z) \equiv \frac{\pi}{\sin \pi \beta}\left[\frac{\mathrm{M}(\alpha, \beta, z)}{\Gamma(1+\alpha-\beta) \Gamma(\beta)}-z^{1-\beta} \frac{\mathrm{M}(1+\alpha-\beta, 2-\beta, z)}{\Gamma(\alpha) \Gamma(2-\beta)}\right]
$$

where $\Gamma(z) \equiv \int_{0}^{\infty} t^{z-1} \mathrm{e}^{-t} \mathrm{~d} t$ is the standard gamma-function. The function $\mathrm{U}$ is also sometimes denoted by $\Psi$.

The Kummer functions are two linearly independent solutions of the differential equation $z F^{\prime \prime}+(\beta-z) F^{\prime}-\alpha F=0$. Their derivatives with respect to $z$ are

$$
\begin{aligned}
& \frac{\partial^{n} \mathrm{M}(\alpha, \beta ; z)}{\partial z^{n}}=\frac{(\alpha)_{n}}{(\beta)_{n}} \mathrm{M}(\alpha+n, \beta+n ; z), \\
& \frac{\partial^{n} \mathrm{U}(\alpha, \beta ; z)}{\partial z^{n}}=(-1)^{n}(\alpha)_{n} \mathrm{U}(\alpha+n, \beta+n ; z),
\end{aligned}
$$

and we have the identities

$$
\mathrm{M}(\alpha, \beta ; z)=\mathrm{e}^{z} \mathrm{M}(\beta-\alpha, \beta ;-z), \quad \mathrm{U}(\alpha, \beta ; z)=z^{1-\beta} \mathrm{U}(1+\alpha-\beta, 2-\beta ; z) .
$$

For the particular choice of parameters, the Kummer functions are reduced to:

$$
\begin{aligned}
\mathrm{M}(0,1 ; z) & =\mathrm{U}(0,1 ; z)=1, \\
\mathrm{M}(1,2 ; z) & =\left(\mathrm{e}^{z}-1\right) z^{-1}, \quad \mathrm{U}(1,2 ; z)=z^{-1}, \\
\left.\frac{\partial \mathrm{M}(\alpha, 1 ; z)}{\partial \alpha}\right|_{\alpha=0} & =\operatorname{chi}(z)+\operatorname{shi}(z)-\gamma-\log (z), \\
\left.\frac{\partial \mathrm{U}(\alpha, 1 ; z)}{\partial \alpha}\right|_{\alpha=0} & =-\log (z),
\end{aligned}
$$

where $\gamma \approx 0.57721$ is the Euler-Mascheroni constant, and $\operatorname{chi}(z)$ and $\operatorname{shi}(z)$ are the hyperbolic cosine and sine integral functions, respectively.

The following asymptotic expansions are valid for the Kummer functions with $\operatorname{Re} z>0$ :

$$
\begin{aligned}
\mathrm{M}(\alpha, \beta ; z) & \sim \frac{\Gamma(\beta) \mathrm{e}^{z} z^{\alpha-\beta}}{\Gamma(\alpha)}+\frac{(-1)^{-\alpha} \Gamma(\beta) z^{-\alpha}}{\Gamma(\beta-\alpha)} & \text { as } \quad z \rightarrow \infty, \\
\mathrm{U}(\alpha, \beta ; z) & \sim z^{-\alpha}+\alpha(\beta-1-\alpha) z^{-\alpha-1} & \text { as } \quad z \rightarrow \infty, \\
\mathrm{M}(\alpha, \beta ; z) & \sim \Gamma(\beta)(\sqrt{\alpha z})^{1-\beta} \mathrm{I}_{\beta-1}(2 \sqrt{\alpha z}) & \text { as } \quad \alpha \rightarrow \infty, \\
\mathrm{U}(\alpha, \beta ; z) & \sim \frac{2}{\Gamma(1+\alpha-\beta)}(\sqrt{\alpha z})^{1-\beta} \mathrm{K}_{\beta-1}(2 \sqrt{\alpha z}) & \text { as } \quad \alpha \rightarrow \infty,
\end{aligned}
$$

where $\mathrm{I}_{\nu}(z)$ and $\mathrm{K}_{\nu}(z)$ are the modified Bessel functions of the first and second kind, respectively. 


\section{References}

[1] P. H. LeBlond And L. A. Mysak, Waves in the ocean, Elsevier Oceanography Series 20, 1978.

[2] C.-S. YIH, Stratified flows, Academic Press, 1980.

[3] L. M. Brekhovskikh and V. V. Goncharov, Mechanics of Continua and Wave Dynamics, Springer, Berlin, 1994.

[4] M.-L. Dubreil-Jacotin, Sur les ondes de type permanent dans les liquides hétérogènes, Accad. Naz. Lincei 6(15):814-819, 1932 (in French).

[5] M.-L. Dubreil-Jacotin, Complément à une note antérieure sur les ondes de type permanent dans les liquides hétérogènes, Rend. Accad. Naz. Lincei 6(21):344-346, 1935 (in French).

[6] M.-L. Dubreil-Jacotin, Sur les théorèmes d'existence relatifs aux ondes permanentes périodiques à deux dimensions dans les liquides hétérogènes, J. Math. Pures Appl. 9(16):184-196, 1937 (in French).

[7] R. R. Long, Some aspects of the flow of stratified fluids. III Continous density gradients. Tellus 7:341-357, 1955.

[8] R. R. Long, On the Boussinesq approximation and its role in the theory of internal waves. Tellus 17:46-52, 1965.

[9] F. Gerstner, Theorie der Wellen samt einer daraus abgeleiteten Theorie der Deichprofile. Ann. Phys. 2:412-445, 1809 (in German).

[10] L. M. Milne-Thomson, Theoretical Hydrodynamics, The Macmillan Co., London, 1938.

[11] D. J. Brown, D. R. Christie, Fully nonlinear solitary waves in continuously stratified incompressible Boussinesq fluids. Phys. Fluids 10(10):2569-2586, 1998.

[12] O. G. Derzho, R. Grimshaw, Solitary waves with a vortex core in a shallow layer of stratified fluid. Phys. Fluids 9(11):3378-3384, 1997.

[13] C.-S. Yin, Stratified flows. Ann. Rev. Fluid Mech. 1:73-110, 1969.

[14] M. Abramowitz, I. A. Stegun, Handbook of Mathematical Functions, Dover, 1965.

[15] E. L. Ince, Ordinary Differential Equations, Dover, 1956.

[16] J. McCowan, On the solitary wave. Phil. Mag. S. 5 32(194):45-58, 1891.

[17] G. G. Stokes, The outskirts of the solitary wave. Mathematical and physical papers, vol. V, Cambridge University Press, 1895.

[18] G. B. Whitham, Linear and Nonlinear Waves, Wiley, 1999. 
[19] E. A. Karabut, An approximation for the highest gravity waves on water of finite depth. J. Fluid Mech., 372:45-70,1998.

[20] M. Yanowitch, Gravity waves in a heterogeneous incompressible fluid. Comm. Pure App. Mat. 15:45-61, 1962.

[21] L. J. Slater, Confluent hypergeometric functions. Cambridge University Press, 1960.

[22] Yu. A. Brychkov, Special functions. CRC Press, 2008. 


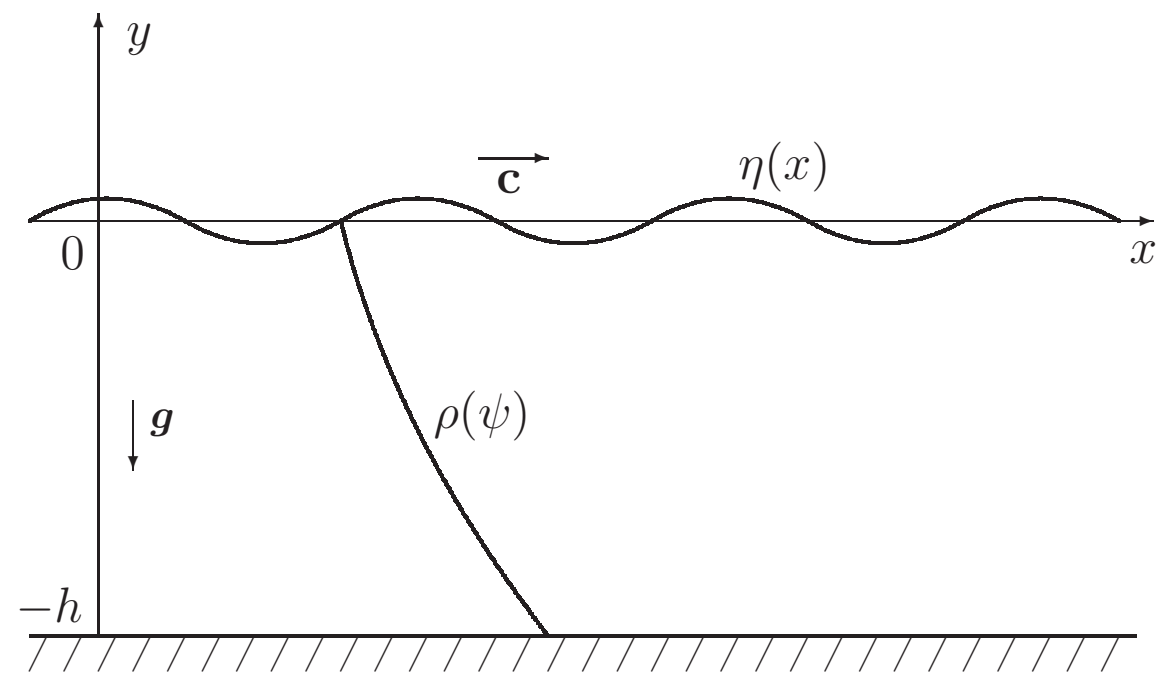

Figure 1: Sketch of the two-dimensional fluid flow in the reference coordinate frame linked with the immovable bottom. Wave of a positive velocity $c$ propagates to the right as shown by the arrow. 


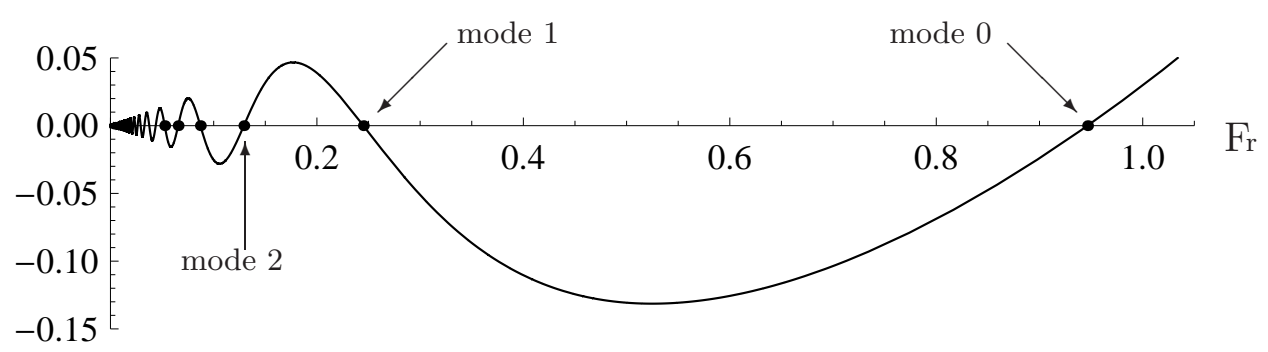

Figure 2: Graphical solution of the dispersion relation (37) with $\varrho=1$ for long waves of infinitesimal amplitude in shallow water. Solid line represents the right-hand side function in Eq. (37); dots are the roots of that equation.

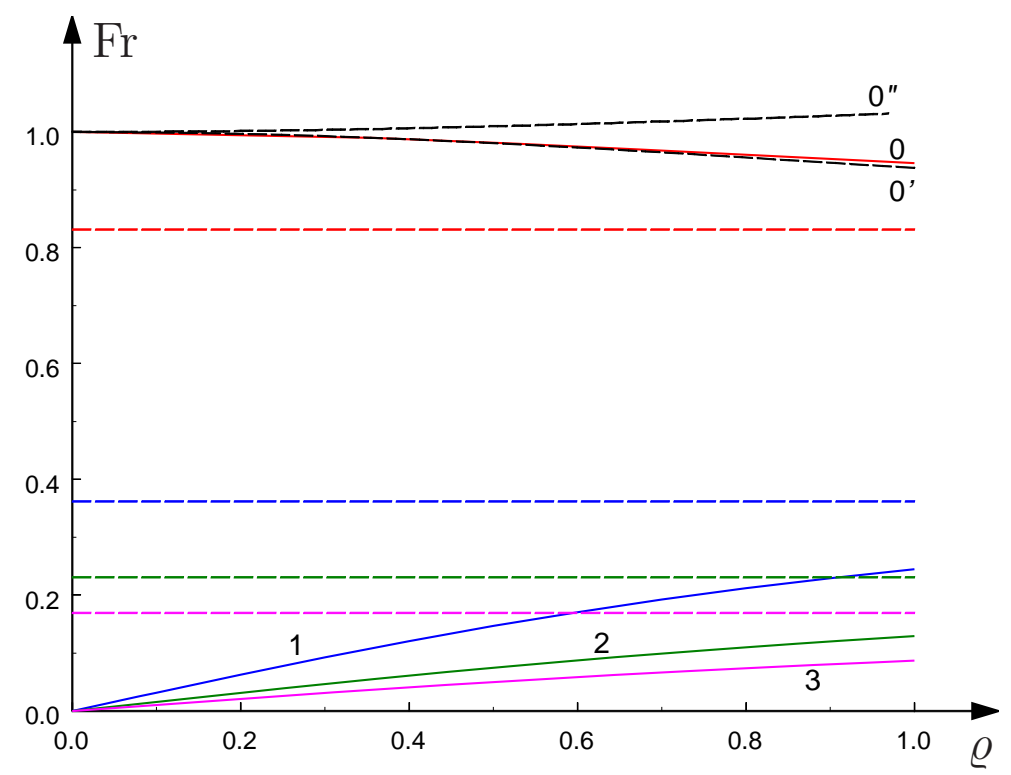

Figure 3: (color online) Froude number against the stratification parameter $\varrho$ for the first four modes of infinitely long waves. The surface mode is labeled by 0 , and first three internal modes are labeled by numbers $1,2,3$. Line $0^{\prime}$ shows the asymptotic dependence for the surface mode when $\varrho \rightarrow 0$, and line $0^{\prime \prime}$ shows the asymptotic dependence for the surface mode when $\mathrm{Fr} \rightarrow 0$. The color dashed horizontal lines show the asymptotic values of corresponding dispersion lines when $\varrho \rightarrow \infty$. 


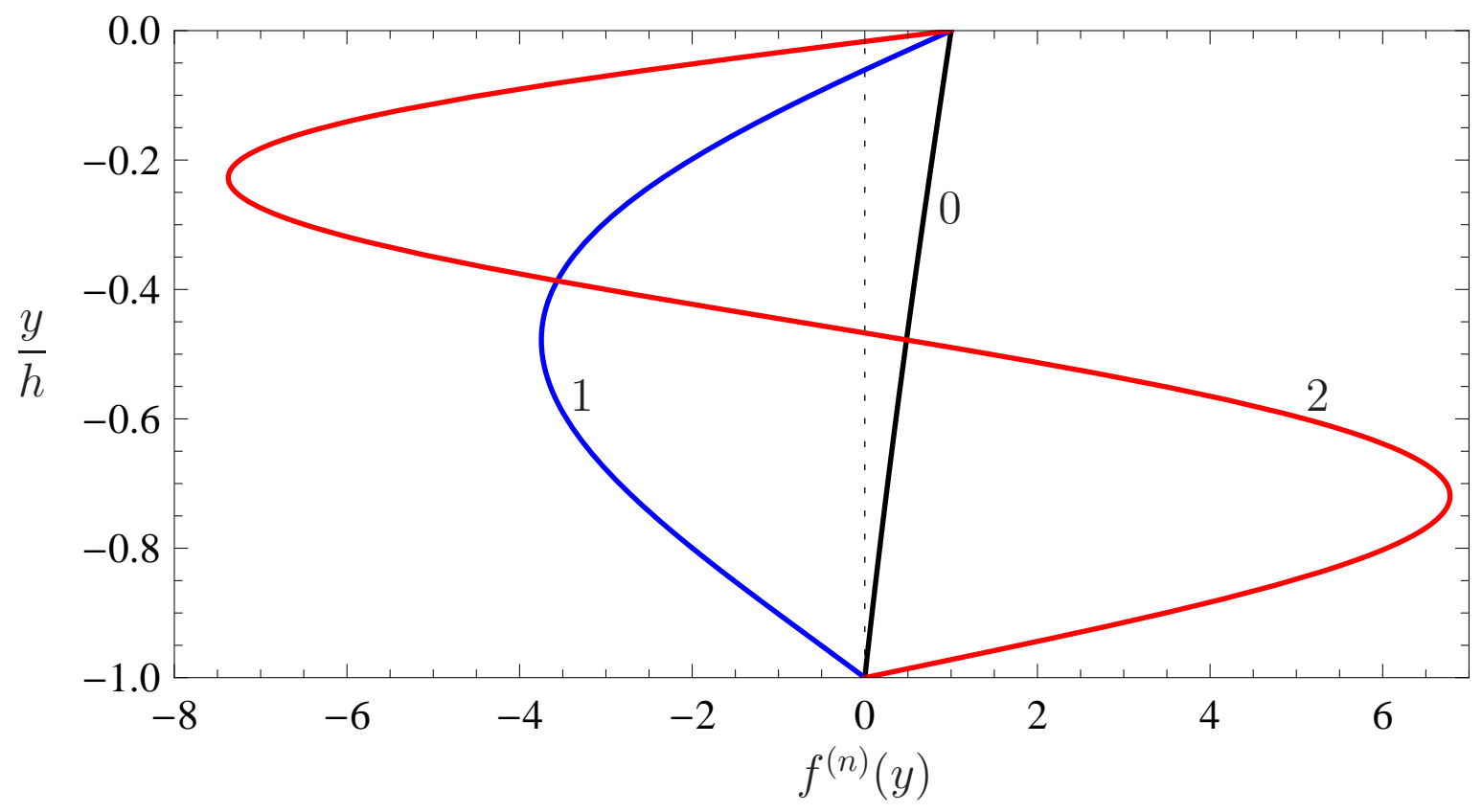

Figure 4: (color online) The structure of the first three modes of infinitely long waves in linear stratification (15) for $\varrho=1$. The numbers in the plots indicate the mode numbers.

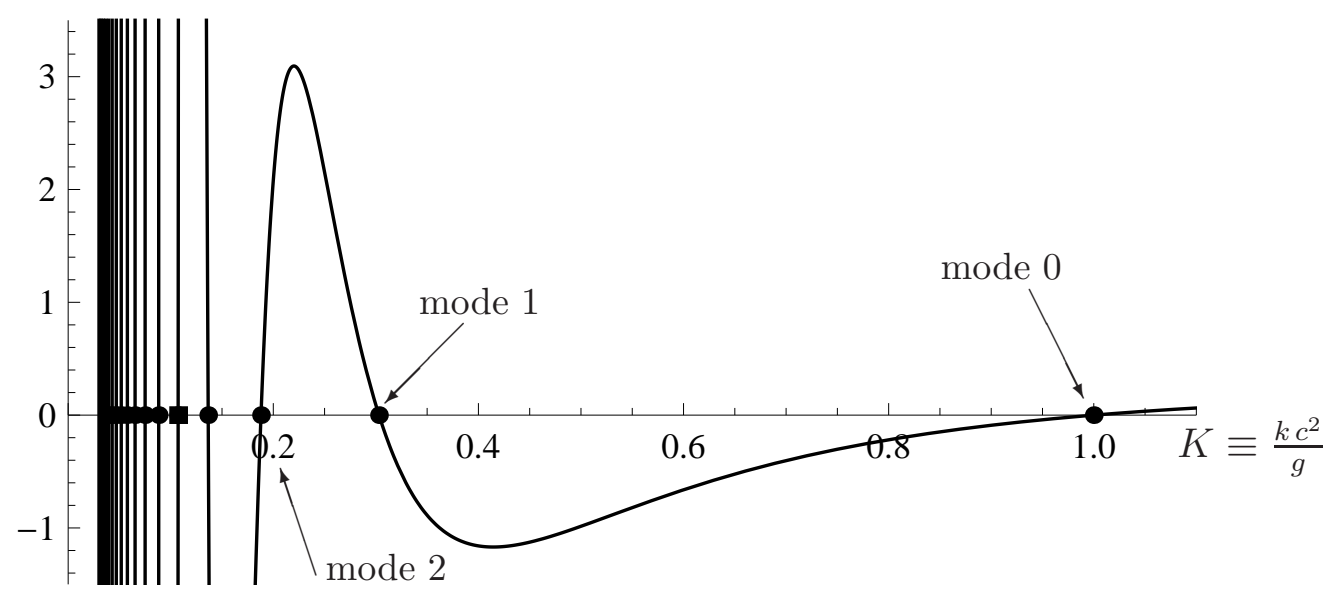

Figure 5: Graphical solution of the dispersion relation (41) for the deep-water limit with $\mathrm{Fd}=1$. 


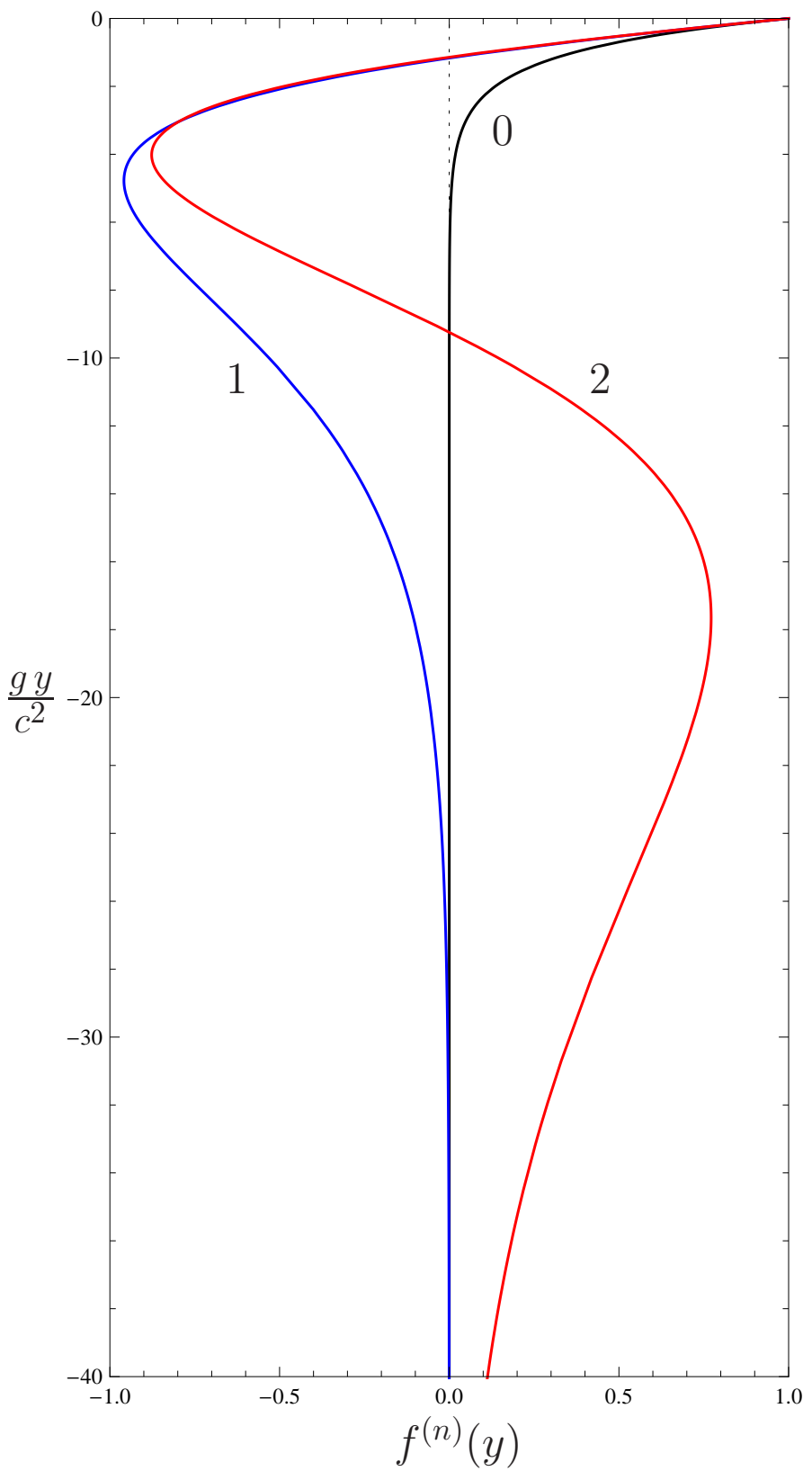

Figure 6: (color online) The first three eigenfunctions $f^{(n)}(y)$ in the case of deep water for $\mathrm{Fd}=1$. a): $n=0\left(K^{(0)}=1\right) ; \quad$ b) $: n=1\left(K^{(1)} \approx 0.303\right) ; \quad$ c) $: n=2\left(K^{(2)} \approx 0.188\right)$. All eigenfunctions are normalized such that $f^{(n)}(0)=1$ [see Eq. (22)]. 